\title{
Hospital capacity during the COVID-19 pandemic
}

\author{
Norihiro Kokudo*, Haruhito Sugiyama \\ National Center for Global Health and Medicine, Tokyo, Japan.
}

\begin{abstract}
The rapid global spread of the COVID-19 pandemic has posed a significant challenge to various countries in terms of the capacity of hospitals to admit and care for patients during the crisis. To estimate hospital capacity during the COVID-19 pandemic, clinicians working in tertiary hospitals around the world were surveyed regarding available COVID-19 hospital statistics. Data were obtained from 8 tertiary centers in 8 countries including the United States, United Kingdom, Switzerland, Turkey, Singapore, India, Pakistan, and Japan. The correlation between the number of patients with COVID-19 per 1 million population $v s$. the maximum number of inpatients with COVID-19 in a representative tertiary hospital in each country was determined, as was the correlation between COVID-19 deaths per 1 million population $v s$. the maximum number of patients with COVID-19 in the intensive care unit (ICU). What was noteworthy was that none of the 8 hospitals reduced emergency room (ER) activity even at the peak of the pandemic although treatment of patients without COVID-19 decreased by $0-70 \%$ depending on the extent of the epidemic. Although various measures are being actively implemented to slow the spread of the virus and reduce the strain on the health care system, the reality is that there are still a significant number of hospitals at risk of being overloaded in the event of a future surge in cases.
\end{abstract}

Keywords: COVID-19, hospital, capacity, ICU, ER

Since the outbreak of COVID-19 in January 2020, the pandemic has struck Japan in 3 waves (Figure 1) (1). The third most recent wave was the largest, and hospital beds for patients with COVID-19 were almost fully occupied in large cities. In Tokyo, the medical system was literally on the verge of collapse. From a global point of view, however, there were relatively few patients with COVID-19 per population in Asia. According to recent global data, there were 3,587 patients with COVID-19 in Japan and 69 deaths per million population. Those numbers were much smaller than numbers in Western countries e.g. the United States (91,537 and 1,667, respectively) and the United Kingdom (62,895 and 1,849, respectively) (2).

The National Center for Global Health and Medicine (NCGM) is one of the 6 National Centers in Japan with a specified mission that includes dealing with infectious disease outbreaks. NCGM Center Hospital is a special function (tertiary) hospital with 700 beds including 4 beds in the high consequence infectious diseases (HCID) unit and 21 negative pressure beds. The NCGM Center Hospital has been expanding its capacity for COVID-19 patients depending on the patient load, with a peak capacity of 70 beds including 8 intensive care unit (ICU) beds (Figure 2). The Hospital had to allocate another $8 \mathrm{ICU}$ beds for critical care patients without COVID-19 to continue functioning as a tertiary general hospital. There are more than 15 tertiary hospitals (most of which are University Hospitals) in the Tokyo metropolitan area, and they allocated a similar number of ICU beds for patients with COVID-19. In total, around 300 ICU beds were allocated, with additional ICU beds allocated in other city hospitals. According to the Tokyo Metropolitan Government, the number of patients with COVID-19 in the ICU (either on a respirator or ECMO) peaked at around 160 (3), and a collapse of the medical system was narrowly avoided.

As COVID-19 spreads rapidly around the country, a major concern has been the ability of hospitals to admit and care for patients (4-8). Many countries initially imposed stay-at-home orders and limited business activity in order to slow the spread of the virus and reduce the strain on health care systems, and these measures have been effective in managing the health crisis. However, there are still a significant number of hospitals at risk of being overloaded in the event of a future surge in cases.

In Western countries where patients with COVID-19 were much more numerous, a number of personal communications among clinicians have reported, say, that more than 200 patients were hospitalized for COVID-19 or that more than 50 patients were treated in the ICU of tertiary hospitals. Since the major of Japanese hospitals cannot sustain the treatment of so 


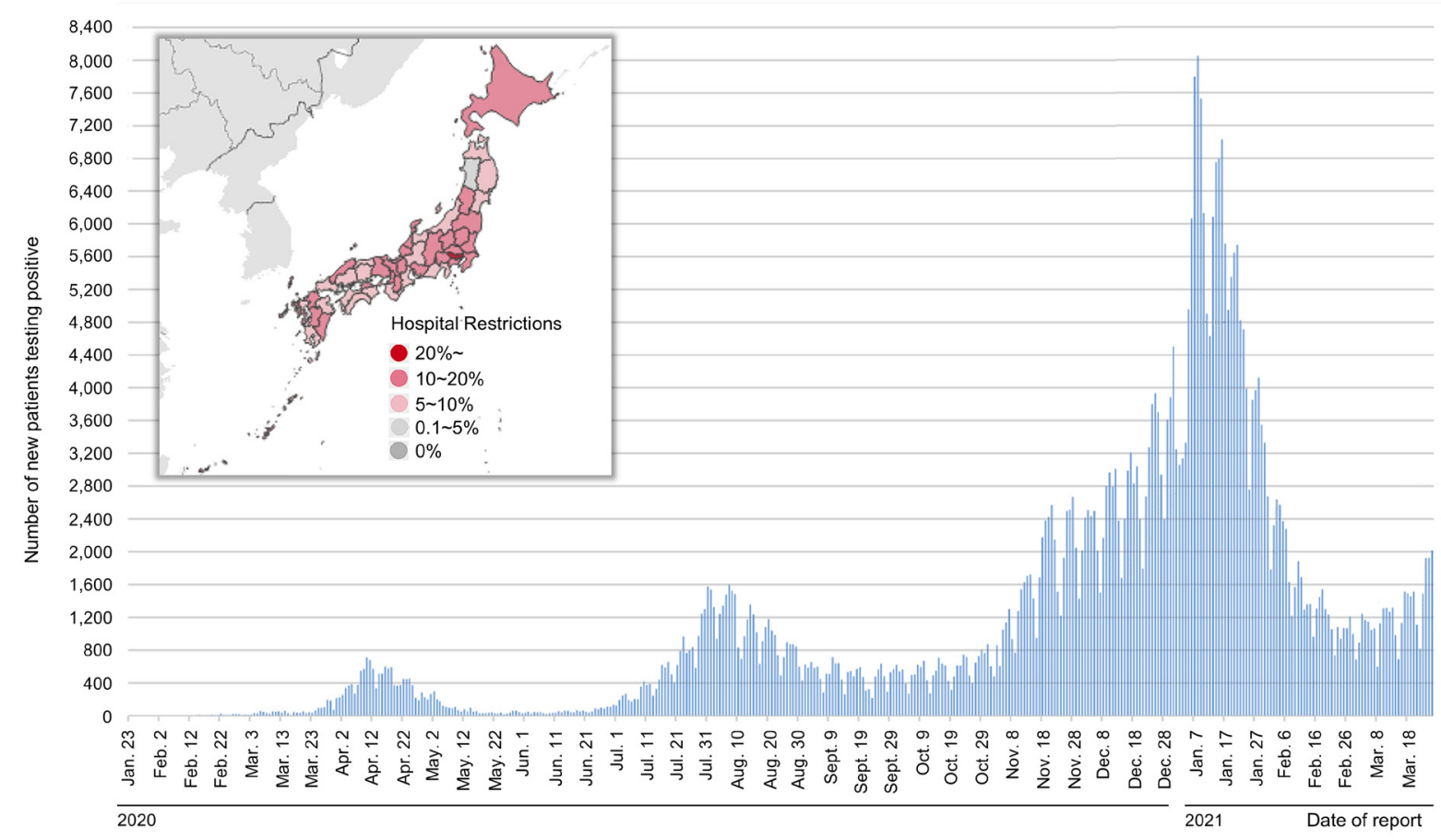

Figure 1. Daily number of newly confirmed COVID-19 cases and status of hospital restrictions in Japan. (Data Source: https://www.mhlw.go.jp/content/10906000/000760545.pdf, https://www.mhlw.go.jp/stf/seisakunitsuite/bunya/0000164708_00001. $h$ tml)

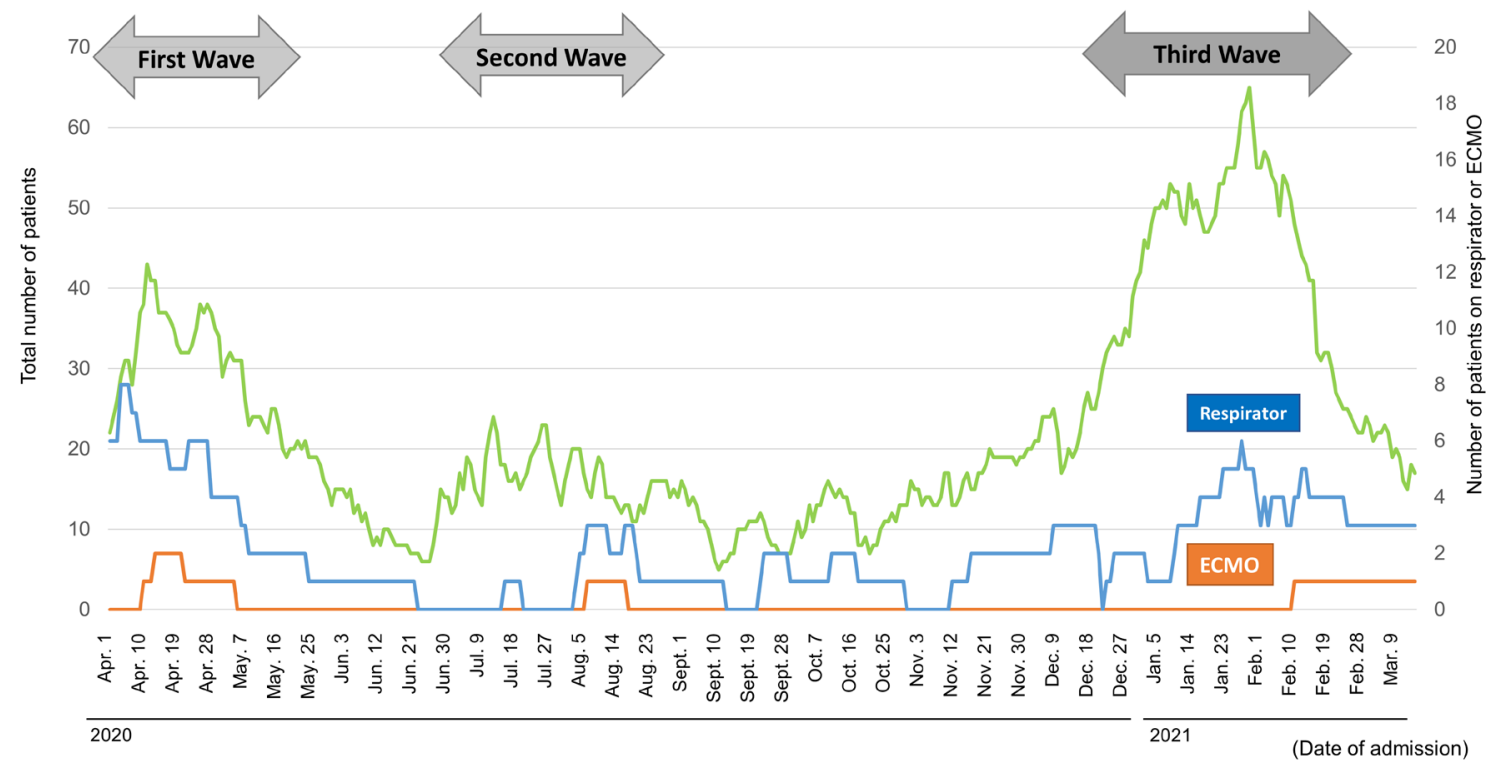

Figure 2. Number of patients with COVID-19 admitted to the NCGM Center Hospital (April 2020 March 2021). NCGM: National Center for Global Health and Medicine.

many patients with COVID-19, the current authors were very curious about the difference in numbers. However, obtaining official COVID-19 hospital statistics is difficult because such data are extremely sensitive and hospital administrators are generally very reluctant to disclose them. Therefore, clinicians working in tertiary hospitals around the world were personally contacted to inquire about available COVID-19 hospital statistics. Admittedly, the data obtained are not official hospital data and some may originate from internal personal communications. This is why all of these hospital data other than those from the NCGM have been kept anonymous except for nationality.

Data were obtained from 8 tertiary centers in 8 

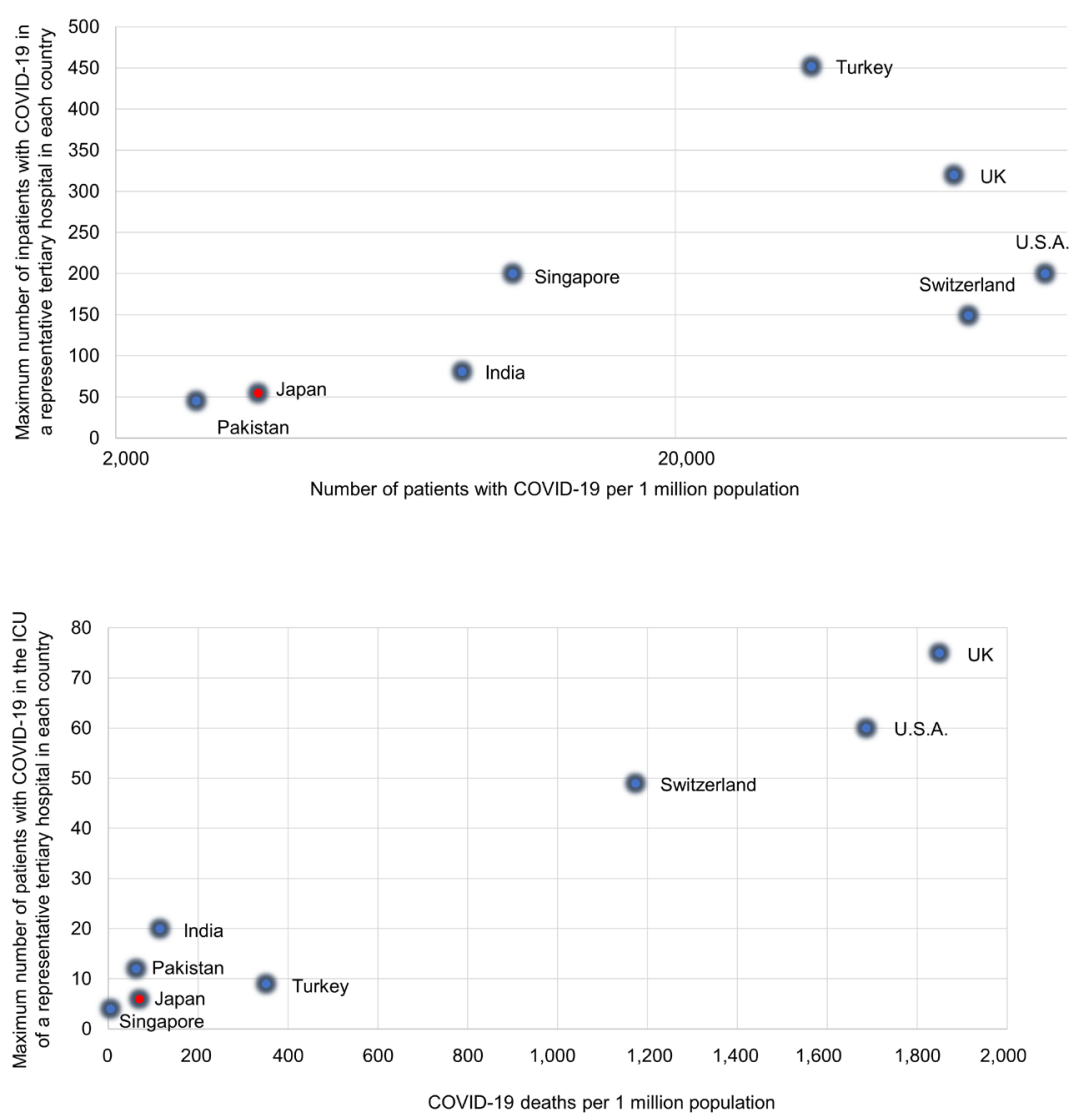

Figure 3. Number of patients with COVID-19 per 1 million population vs. maximum number of inpatients with COVID-19 in a representative tertiary hospital in each country.
Figure 4. COVID-19 death per 1 million population vs. maximum number of patients with COVID-19 in the ICU of a representative tertiary hospital in each country. countries. Figure 3 shows the correlation between the number of patients with COVID-19 per 1 million population $v s$. the maximum number of inpatients with COVID-19 in a representative tertiary hospital in each country. There was a roughly logarithmic correlation and Japan (the NCGM Center Hospital) is located in the bottom left of the chart. Figure 4 shows the correlation between COVID-19 deaths per 1 million population $v s$. the maximum number of patients with COVID-19 in the ICU. Asian centers including the NCGM Center Hospital were located in the bottom left of the chart and Western countries in the top right. Although the correlations in Figure 3 and Figure 4 are very rough ones, relatively few medical resources were deployed in Japan and the rest of Asia thanks to relatively small numbers of patients. However, expansion of hospital capacity in the event of a worsening epidemic may be difficult because of limited medical personnel in Japan. According to this survey, there were 3 to 5 times as many doctors in Western hospitals as there were in Japanese hospitals and 2-3 times as many nurses. Japan may urgently need the capacity to deal with a surge. What was noteworthy was none of the 8 hospitals reduced emergency room (ER) activity even at the peak of the pandemic although treatment of patients without COVID-19 decreased by $0-70 \%$ depending on the extent of the epidemic.

A point that need not be mentioned is that data from a single hospital cannot represent an entire country. Given the difficulty in obtaining such data, personal communications may provide useful information for formulating a strategy in the event of a pandemic. Although various measures are being actively implemented to slow the spread of the virus and reduce the strain on the health care system, the reality is that there are still a significant number of hospitals at risk of being overloaded in the event of a future surge in cases.

\section{Acknowledgements}

The authors would like to sincerely thank all of the clinicians who were involved in this study. Specifically, the authors would like to thank the following doctors for their assistance with the survey: Harshitha Mergey Devender, Ender Dulundu, Ghumza Farooq, Nermin Halkic, Masatoshi Hotta, Akiho Sugita, Hideaki Yano, and Kosuke Yasukawa (in alphabetical order).

\section{Funding: None.}

Conflict of Interest: The authors have no conflicts of interest to disclose.

\section{References}

1. Ministry of Health, Labor, and Welfare. Trends in domestic outbreaks of COVID-19. https://www.mhlw. go.jp/content/10906000/000760545.pdf (accessed March 27, 2021) (in Japanese) 
2. Worldometer. COVID-19 Coronavirus pandemic. https:// www.worldometers.info/coronavirus (accessed March 27, 2021)

3. Bureau of Social Welfare and Public Health. Status of the Infection. https://www.fukushihoken.metro.tokyo. lg.jp/iryo/kansen/monitoring.files/0325gurafumatome.pdf (accessed March 27, 2021) (in Japanese)

4. McCabe R, Schmit N, Christen P, D'Aeth JC, Løchen A, Rizmie D, Nayagam S, Miraldo M, Aylin P, Bottle A, Perez-Guzman PN, Ghani AC, Ferguson NM, White PJ, Hauck K. Adapting hospital capacity to meet changing demands during the COVID-19 pandemic. BMC Med. 2020; 18:329.

5. Litton E, Bucci T, Chavan S, Ho YY, Holley A, Howard G, Huckson S, Kwong P, Millar J, Nguyen N, Secombe $\mathrm{P}$, Ziegenfuss M, Pilcher D. Surge capacity of intensive care units in case of acute increase in demand caused by COVID-19 in Australia. Med J Aust. 2020; 212:463-467.

6. Carenzo L, Costantini E, Greco M, Barra FL, Rendiniello V, Mainetti M, Bui R, Zanella A, Grasselli G, Lagioia M, Protti A, Cecconi M. Hospital surge capacity in a tertiary emergency referral centre during the COVID-19 outbreak in Italy. Anaesthesia. 2020; 75:928-934.

7. Aziz S, Arabi YM, Alhazzani W, et al. Managing ICU surge during the COVID-19 crisis: Rapid guidelines. Intensive Care Med. 2020; 46:1303-1325.

8. Rossman H, Meir T, Somer J, Shilo S, Gutman R, Ben Arie A, Segal E, Shalit U, Gorfine M. Hospital load and increased COVID-19 related mortality in Israel. Nat Commun. 2021; 12:1904.

Received March 28, 2021; Accepted April 8, 2021.

Released online in J-STAGE as advance publication April 11, 2021.

*Address correspondence to:

Norihiro Kokudo, President, National Center for Global Health and Medicine, 1-21-1 Toyama, Shinjuku-ku, Tokyo 162-8655, Japan.

E-mail: nkokudo@hosp.ncgm.go.jp 